\title{
ROBUST MEASURE TRANSFORMED MUSIC FOR DOA ESTIMATION
}

\author{
Koby Todros \\ Ben-Gurion University of the Negev
}

\author{
Alfred O. Hero
}

University of Michigan

\begin{abstract}
In this paper, we introduce a new framework for robust multiple signal classification (MUSIC). The proposed framework, called robust measure-transformed (MT) MUSIC, is based on applying a transform to the probability distribution of the received signals, i.e., transformation of the probability measure defined on their observation space. In robust MT-MUSIC, the sample covariance is replaced by the empirical MT-covariance. By judicious choice of the transform we show that: (1) the resulting empirical MT-covariance is Brobust, with bounded influence function that takes negligible values for large norm outliers, and (2) under the assumption of spherical compound Gaussian noise, the noise subspace can be determined from the eigendecomposition of the MT-covariance. The proposed approach is illustrated for direction-of-arrival (DOA) estimation in a simulation example that shows its advantages as compared to other robust MUSIC generalizations.
\end{abstract}

Index Terms - Array processing, DOA estimation, probability measure transform, robust estimation, signal subspace estimation.

\section{INTRODUCTION}

The multiple signal classification (MUSIC) algorithm [1], [2] is a well established technique for estimating direction-of-arrivals (DOAs) of signals impinging on an array of sensors. It operates by finding DOAs with corresponding array steering vectors that have minimal projections onto the empirical noise subspace, whose spanning vectors are obtained via eigendecomposition of the sample covariance matrix (SCM) of the array outputs.

In the presence of outliers, possibly caused by heavy-tailed impulsive noise, the SCM poorly estimates the covariance of the array outputs, resulting in unreliable DOAs estimates. In order to overcome this limitation, several MUSIC generalizations have been proposed in the literature that replace the SCM with robust association or scatter matrix estimators, for which the empirical noise subspace can be determined from their eigendecomposition.

Under the assumption that the signal and noise components are jointly $\alpha$-stable processes [3], it was proposed in [4] to replace the SCM with empirical covariation matrices that involve fractional lower-order statistics. Although $\alpha$-stable processes are appropriate for modeling impulsive noise [5], the assumption that the signal and noise components are jointly $\alpha$-stable is not practical. In [6], a less restrictive approach considering circular signals contaminated by additive $\alpha$-stable noise was developed that replaces the SCM with matrices comprised of empirical fractional-lower-order-moments. Although this approach is less restrictive than the one proposed in [4], violation of the signals circularity assumption, e.g., by applying BPSK signals, results in poor DOA estimation performance [6].

This research was partially supported by NSF grant CCF-1217880 and ARO grant W911NF-11-1-0391.
In [7] a different MUSIC generalization was proposed that replaces the SCM with empirical sign or rank covariances. Using only the assumption of spherically symmetric noise, it was shown that convergent estimates of the noise subspace can be obtained from their eigendecomposition. The empirical sign and rank covariance matrices have bounded influence functions [8], in other words they are B-robust. However, it can be shown that the Frobenius norms of their influence functions do not reject large outliers, i.e., they do not approach zero as the magnitude of the outlier approaches infinity. Furthermore, the emprical sign and rank covariance matrices have influence functions with constant Frobenius norms for spherically symmetric distributions.

In [9], robust M-estimators of scatter [10], [11], such as the maximum-likelihood, Huber's [11], and Tyler's [12] M-estimators, extended to complex elliptically symmetric (CES) distributions, were proposed as alternatives to the SCM. Under the class of CES distributions having finite second-order moments, these estimators provide consistent estimation of the covariance up to a positive scalar, resulting in consistent estimation of the noise subspace. Although this approach can provide robustness against outliers with negligible loss in efficiency when the observations are normally distributed, it may suffer from the following drawbacks. First, when the observations are not elliptically distributed, M-estimators may loose the consistency relation to the covariance matrix [13], which may lead to poor estimation of the noise subspace. Second, M-estimators of scatter are computed using iterative fixed-point algorithm that converges to a unique solution under some regularity conditions. Each iteration involves matrix inversion which may be computationally demanding in high dimensions, or unstable when the scatter matrix is close to singular. Moreover, although the influence functions of M-estimators may be bounded, they may not behave well for large norm outliers which can affect the estimation performance. Indeed, similarly to the method of [7], Tyler's scatter M-estimator does not reject large outliers and its influence function has constant Frobenius norm for spherically symmetric distributions.

In this paper, we introduce a new non-iterative framework for robust MUSIC. The proposed framework, called robust measuretransformed MUSIC (MT-MUSIC), is inspired by a measure transformation approach that was recently applied to canonical correlation analysis [14] and independent component analysis [15]. Robust MT-MUSIC is based on applying a transform to the probability distribution of the data. The proposed transform is structured by a nonnegative function, called the MT-function, and maps the probability distribution into a set of new probability measures on the observation space. By modifying the MT-function, classes of measure transformations can be obtained that have different useful properties. Under the proposed transform we define the measure-transformed (MT) covariance and derive its strongly consistent estimate, which is also shown to be Fisher consistent [16]. Robustness of the empirical MTcovariance is established in terms of boundedness of its influence function. A sufficient condition on the MT-function that guarantees 
B-robustness of the empirical MT-covariance is obtained.

In robust MT-MUSIC, the SCM is replaced by the empirical MTcovariance. The MT-function is selected such that the resulting empirical MT-covariance is B-robust, and the noise subspace can be determined from the eigendecomposition of the MT-covariance. By modifying the MT-function such that these conditions are satisfied a class of robust MT-MUSIC algorithms can be obtained.

Selection of the MT-function under the family of zero-centered Gaussian functions, parameterized by a scale parameter, results in a new algorithm called Gaussian MT-MUSIC. We show that the empirical Gaussian MT-covariance is B-robust with influence function that approaches zero as the outlier magnitude approaches infinity. Under the additional assumption that the noise component has a spherical compound Gaussian distribution, we show that the noise subspace can be determined from the eigendecomposition of Gaussian MT-covariance. The family of compound Gaussian distributions encompasses common heavy-tailed distributions, such as the Cauchy distribution, and has been widely used for modeling radar clutter [17]-[20]. We propose a data-driven procedure for selecting the Gaussian MT-function scale parameter. This procedure has the property that it prevents significant transform-domain Fisher-information loss when the observations are normally distributed.

The Gaussian MT-MUSIC is evaluated by simulation to illustrate its advantages relative to other robust MUSIC algorithms.

The paper is organized as follows. In section 2, the robust MTMUSIC framework is developed. In Section 3, the Gaussian MTMUSIC algorithm is derived from the proposed framework. This algorithm is illustrated in a simulation example in Section 4. In Section 5, the main points of this contribution are summarized. Proofs for the propositions stated throughout the paper will be provided in the full length journal version.

\section{ROBUST MEASURE-TRANSFORMED MUSIC}

In this section, the robust MT-MUSIC procedure is presented. First, the sensor array model is introduced. Second, a general transformation on probability measures is established. Under the proposed transform, we define the MT-covariance matrix and derive its strongly consistent estimate. Robustness of the empirical MTcovariance is studied by analyzing its influence function. Finally, based on the assumed array model, we propose a robust MT-MUSIC procedure that replaces the SCM with the empirical MT-covariance of the received signals.

\subsection{Array model}

Consider an array of $p$ sensors that receive signals generated by $q<p$ narrowband incoherent far-field point sources with distinct azimuthal DOAs $\theta_{1}, \ldots, \theta_{q}$. Under this model, the array output satisfies [2]:

$$
\mathbf{X}_{n}=\mathbf{A}(\boldsymbol{\theta}) \mathbf{S}_{n}+\mathbf{W}_{n},
$$

where $n \in \mathbb{N}$ is a discrete time index, $\mathbf{X}_{n} \in \mathbb{C}^{p}$ is the vector of received signals, $\mathbf{S}_{n} \in \mathbb{C}^{q}$ is a zero-mean latent random vector, comprised of the emitted signals, with non-singular covariance, and $\mathbf{W}_{n} \in \mathbb{C}^{p}$ is an additive zero-mean spatially white noise. The ma$\operatorname{trix} \mathbf{A}(\boldsymbol{\theta}) \triangleq\left[\mathbf{a}\left(\theta_{1}\right), \ldots, \mathbf{a}\left(\theta_{q}\right)\right] \in \mathbb{C}^{p \times q}$ is the array steering matrix, where $\boldsymbol{\theta} \triangleq\left[\theta_{1}, \ldots, \theta_{q}\right]^{T}$ and $\mathbf{a}(\theta)$ is the steering vector of the array toward direction $\theta$. We assume that the array is unambiguous, i.e., any collection of $p$ steering vectors corresponding to distinct DOAs forms a linearly independent set. Therefore, $\mathbf{A}(\boldsymbol{\theta})$ has full column rank, and identification of its column vectors is equivalent to the problem of identifying the DOAs. We also assume that $\mathbf{S}_{n}$ and $\mathbf{W}_{n}$ are statistically independent and first-order stationary. To simplify notation, the time index $n$ will be omitted in the sequel except where noted.

\subsection{Probability measure transform}

We define the measure space $\left(\mathcal{X}, \mathcal{S}_{\mathcal{X}}, P_{\mathbf{X}}\right)$, where $\mathcal{X}$ is an observation space of a random vector $\mathbf{X} \in \mathbb{C}^{p}, \mathcal{S}_{\mathcal{X}}$ is a $\sigma$-algebra over $\mathcal{X}$, and $P_{\mathbf{X}}$ is a probability measure on $\mathcal{S}_{\mathcal{X}}$.

Definition 1. Given a non-negative function $u: \mathbb{C}^{p} \rightarrow \mathbb{R}_{+}$satisfying $0<\mathrm{E}\left[u(\mathbf{X}) ; P_{\mathbf{X}}\right]<\infty$, where $\mathrm{E}\left[\cdot ; P_{\mathbf{X}}\right]$ denotes the expectation under $P_{\mathbf{X}}$, a transform on $P_{\mathbf{X}}$ is defined via the relation:

$$
Q_{\mathbf{X}}^{(u)}(A) \triangleq \mathrm{T}_{u}\left[P_{\mathbf{X}}\right](A)=\int_{A} \varphi_{u}(\mathbf{x}) d P_{\mathbf{X}}(\mathbf{x}),
$$

where $A \in \mathcal{S}_{\mathcal{X}}, \mathrm{x} \in \mathcal{X}$, and

$$
\varphi_{u}(\mathbf{x}) \triangleq u(\mathbf{x}) / \mathrm{E}\left[u(\mathbf{X}) ; P_{\mathbf{X}}\right] .
$$

The function $u(\cdot)$ is called the MT-function.

Proposition 1 (Properties of the transform). Let $Q_{\mathrm{x}}^{(u)}$ be defined by relation (2). Then

1. $Q_{\mathrm{X}}^{(u)}$ is a probability measure on $\mathcal{S}_{\mathcal{X}}$.

2. $Q_{\mathrm{X}}^{(u)}$ is absolutely continuous w.r.t. $P_{\mathbf{X}}$, with Radon-Nikodym derivative [21]:

$$
d Q_{\mathbf{X}}^{(u)}(\mathbf{x}) / d P_{\mathbf{X}}(\mathbf{x})=\varphi_{u}(\mathbf{x}) .
$$

3. Assume that the MT-function $u(\cdot)$ is strictly positive, and let $\mathrm{g}: \mathcal{X} \rightarrow \mathbb{C}^{m}$ denote an integrable function over $\mathcal{X}$. If the covariance of $\mathbf{g}(\mathbf{X})$ under $P_{\mathbf{X}}$ is non-singular, then it is nonsingular under the transformed probability measure $Q_{\mathrm{X}}^{(u)}$.

4. Assume that the MT-function $u(\cdot)$ is Gaussian. The probability measure $P_{\mathbf{X}}$ is proper complex normal if and only if $Q_{\mathbf{X}}^{(u)}$ is proper complex normal.

\subsection{The measure-transformed covariance}

According to (4) the covariance of $\mathbf{X}$ under $Q_{\mathbf{X}}^{(u)}$ is given by

$$
\boldsymbol{\Sigma}_{\mathbf{X}}^{(u)}=\mathrm{E}\left[\mathbf{X X}^{H} \varphi_{u}(\mathbf{X}) ; P_{\mathbf{X}}\right]-\boldsymbol{\mu}_{\mathbf{X}}^{(u)} \boldsymbol{\mu}_{\mathbf{X}}^{(u) H},
$$

where $\boldsymbol{\mu}_{\mathbf{X}}^{(u)} \triangleq \mathrm{E}\left[\mathbf{X} \varphi_{u}(\mathbf{X}) ; P_{\mathbf{X}}\right]$ is the expectation of $\mathbf{X}$ under $Q_{\mathbf{X}}^{(u)}$. Equation (5) implies that $\boldsymbol{\Sigma}_{\mathbf{X}}^{(u)}$ is a weighted covariance matrix of $\mathbf{X}$ under $P_{\mathbf{X}}$, with weighting function $\varphi_{u}(\cdot)$. Hence, $\boldsymbol{\Sigma}_{\mathbf{X}}^{(u)}$ can be estimated using only samples from the distribution $P_{\mathbf{X}}$. By modifying the MT-function $u(\cdot)$, such that the condition in definition 1 is satisfied, the MT-covariance matrix under $Q_{\mathrm{X}}^{(u)}$ is modified. In particular, by choosing $u(\mathbf{x}) \equiv 1$, we have $Q_{\mathbf{x}}^{(u)}=P_{\mathbf{X}}$, for which the standard covariance matrix $\boldsymbol{\Sigma}_{\mathbf{X}}$ is obtained.

Proposition 2 (Strongly consistent estimate of the MT-covariance). Let $\mathbf{X}_{n}, n=1, \ldots, N$ denote a sequence of i.i.d. samples from $P_{\mathbf{X}}$, and define the empirical covariance estimate

$$
\hat{\boldsymbol{\Sigma}}_{\mathbf{X}}^{(u)} \triangleq \sum_{n=1}^{N} \mathbf{X}_{n} \mathbf{X}_{n}^{H} \hat{\varphi}_{u}\left(\mathbf{X}_{n}\right)-\hat{\boldsymbol{\mu}}_{\mathbf{x}}^{(u)} \hat{\boldsymbol{\mu}}_{\mathbf{x}}^{(u) H},
$$


where $\hat{\boldsymbol{\mu}}_{\mathbf{X}}^{(u)} \triangleq \sum_{n=1}^{N} \mathbf{X}_{n} \hat{\varphi}_{u}\left(\mathbf{X}_{n}\right)$, and $\hat{\varphi}_{u}\left(\mathbf{X}_{n}\right) \triangleq \frac{u\left(\mathbf{X}_{n}\right)}{\sum_{n=1}^{N} u\left(\mathbf{X}_{n}\right)}$. If $\mathrm{E}\left[\|\mathbf{X}\|_{2}^{2} u(\mathbf{X}) ; P_{\mathbf{X}}\right]<\infty$, then $\hat{\mathbf{\Sigma}}_{\mathbf{X}}^{(u)} \rightarrow \mathbf{\Sigma}_{\mathbf{X}}^{(u)}$ almost surely as $N \rightarrow \infty$.

Note that for $u(\mathbf{X}) \equiv 1$ the estimator $\frac{N}{N-1} \hat{\mathbf{\Sigma}}_{\mathbf{X}}^{(u)}$ reduces to the standard unbiased SCM. Also notice that $\hat{\boldsymbol{\Sigma}}_{\mathbf{X}}^{(u)}$ can be written as a statistical functional $\Psi_{\mathrm{X}}^{(u)}[\cdot]$ of the empirical probability measure $\hat{P}_{\mathbf{X}} \triangleq \frac{1}{N} \sum_{n=1}^{N} \delta_{\mathbf{x}_{n}}$, where $\delta_{\mathbf{x}_{n}}$ is the Dirac probability measure at $\mathbf{X}_{n}$, i.e.,

$\hat{\mathbf{\Sigma}}_{\mathbf{X}}^{(u)}=\frac{\mathrm{E}\left[\mathbf{X X} \mathbf{X}^{H} u(\mathbf{X}) ; \hat{P}_{\mathbf{X}}\right]}{\mathrm{E}\left[u(\mathbf{X}) ; \hat{P}_{\mathbf{X}}\right]}-\boldsymbol{\eta}_{\mathbf{X}}^{(u)}\left[\hat{P}_{\mathbf{X}}\right] \boldsymbol{\eta}_{\mathbf{X}}^{(u) H}\left[\hat{P}_{\mathbf{X}}\right] \triangleq \mathbf{\Psi}_{\mathbf{X}}^{(u)}\left[\hat{P}_{\mathbf{X}}\right]$,

where $\boldsymbol{\eta}_{\mathbf{X}}^{(u)}\left[\hat{P}_{\mathbf{X}}\right] \triangleq \mathrm{E}\left[\mathbf{X} u(\mathbf{X}) ; \hat{P}_{\mathbf{X}}\right] / \mathrm{E}\left[u(\mathbf{X}) ; \hat{P}_{\mathbf{X}}\right]$. By (3), (5) and (7), when $\hat{P}_{\mathbf{X}}$ is replaced by $P_{\mathbf{X}}$ we have $\Psi_{\mathbf{X}}^{(u)}\left[P_{\mathbf{X}}\right]=\boldsymbol{\Sigma}_{\mathbf{X}}^{(u)}$, which implies that $\hat{\boldsymbol{\Sigma}}_{\mathbf{X}}^{(u)}$ is Fisher consistent [16].

\subsection{Robustness of the empirical MT-covariance}

Here, we study the robustness of the empirical MT-covariance $\hat{\boldsymbol{\Sigma}}_{\mathbf{X}}^{(u)}$ using its influence function [8]. Define the probability measure $P_{\epsilon} \triangleq$ $(1-\epsilon) P_{\mathbf{X}}+\epsilon \delta_{\mathbf{y}}$, where $0 \leq \epsilon \leq 1, \mathbf{y} \in \mathbb{C}^{p}$, and $\delta_{\mathbf{y}}$ is the Dirac probability measure at $\mathbf{y}$. The influence function of a Fisher consistent estimator with statistical functional $\mathrm{H}[\cdot]$ at probability distribution $P_{\mathrm{X}}$ is defined as [8]:

$$
\mathrm{IF}_{\mathrm{H}}\left(\mathbf{y} ; P_{\mathbf{X}}\right) \triangleq \lim _{\epsilon \rightarrow 0} \frac{\mathrm{H}\left[P_{\epsilon}\right]-\mathrm{H}\left[P_{\mathbf{X}}\right]}{\epsilon}=\left.\frac{\partial \mathrm{H}\left[P_{\epsilon}\right]}{\partial \epsilon}\right|_{\epsilon=0} .
$$

The influence function describes the effect on the estimator of an infinitesimal contamination at the point $\mathbf{y}$. An estimator is said to be B-robust if its influence function is bounded. Using (7) and (8) one can verify that the influence function of $\hat{\boldsymbol{\Sigma}}_{\mathbf{X}}^{(u)}$ is given by

$$
\mathrm{IF}_{\Psi_{\mathbf{x}}^{(u)}}\left(\mathbf{y} ; P_{\mathbf{X}}\right)=\frac{u(\mathbf{y})\left[\left(\mathbf{y}-\boldsymbol{\mu}_{\mathbf{X}}^{(u)}\right)\left(\mathbf{y}-\boldsymbol{\mu}_{\mathbf{X}}^{(u)}\right)^{H}-\boldsymbol{\Sigma}_{\mathbf{X}}^{(u)}\right]}{\mathrm{E}\left[u(\mathbf{X}) ; P_{\mathbf{X}}\right]} .
$$

The following proposition states a sufficient condition for boundedness of (9). This condition is satisfied for the Gaussian MT-function proposed in Section 3.

Proposition 3. The influence function (9) is bounded if the MTfunction $u(\mathbf{y})$ and the product $u(\mathbf{y})\|\mathbf{y}\|_{2}^{2}$ are bounded over $\mathbb{C}^{p}$.

\subsection{The robust MT-MUSIC procedure}

In robust MT-MUSIC the measure transformation (2) is applied to the probability distribution $P_{\mathbf{X}}$ of the array output $\mathbf{X}_{n}$ (1). we propose selecting the MT-function $u(\cdot)$ such that: (1) the resulting empirical MT-covariance $\hat{\boldsymbol{\Sigma}}_{\mathbf{X}}^{(u)}$ is B-robust, and (2) the $p-q$ eigenvectors of $\boldsymbol{\Sigma}_{\mathbf{X}}^{(u)}$ corresponding to its smallest eigenvalues span the null-space of $\mathbf{A}^{H}(\boldsymbol{\theta})$, also called the noise subspace. Let $\hat{\mathbf{V}}^{(u)} \in$ $\mathbb{C}^{p \times(p-q)}$ denote the matrix comprised of $p-q$ eigenvectors of $\hat{\boldsymbol{\Sigma}}_{\mathrm{X}}^{(u)}$ corresponding to its smallest eigenvalues. The DOAs are estimated by finding the $q$ maxima of the measure-transformed pseudospectrum:

$$
\hat{P}^{(u)}(\theta) \triangleq\left\|\hat{\mathbf{V}}^{(u) H} \mathbf{a}(\theta)\right\|^{-2} .
$$

By modifying the MT-function $u(\cdot)$ such that the stated conditions are satisfied a family of robust MT-MUSIC algorithms can be obtained. A particular choice of MT-function leading to the Gaussian MT-MUSIC algorithm is discussed in the following section.

\section{THE GAUSSIAN MT-MUSIC}

In this section, we parameterize the MT-function $u(\cdot ; \tau)$, with scale parameter $\tau \in \mathbb{R}_{+}^{*}$ under the Gaussian family of functions centered at the origin. This results in a B-robust empirical MT-covariance matrix. Under the assumption of spherical compound Gaussian noise, we show that the noise subspace can be determined from the eigendecomposition of the MT-covariance. Choice of the scale parameter $\tau$ is also discussed.

\subsection{The Gaussian MT-function}

We define the Gaussian MT-function $u_{\mathrm{G}}(\cdot ; \cdot)$ as

$$
u_{\mathrm{G}}(\mathbf{x} ; \tau) \triangleq\left(\pi \tau^{2}\right)^{-p} \exp \left(-\|\mathbf{x}\|_{2}^{2} / \tau^{2}\right), \quad \tau \in \mathbb{R}_{+}^{*} .
$$

One can verify that $u_{\mathrm{G}}(\cdot ; \cdot)$ satisfies the condition in proposition 3, resulting in a B-robust empirical Gaussian MT-covariance $\hat{\boldsymbol{\Sigma}}_{\mathbf{X}}^{\left(u_{\mathrm{G}}\right)}(\tau)$. Using (9) and (11) it can be shown that the corresponding influence function satisfies $\left\|\mathrm{IF}_{\Psi_{\mathbf{x}}^{\left(u_{\mathrm{G}}\right)}}\left(\mathbf{y} ; P_{\mathbf{X}}\right)\right\|_{\text {Fro }} \rightarrow 0$ as $\|\mathbf{y}\|_{2} \rightarrow \infty$, where $\|\cdot\|_{\text {Fro denotes the Frobenius norm, i.e., }}$ unlike the SCM and other robust covariance approaches, the empirical Gaussian MT-covariance rejects large outliers. This property is illustrated in Fig. 1 for a bivariate standard complex normal distribution, as compared to the empirical sign-covariance, Tyler's scatter M-estimator, and the SCM.

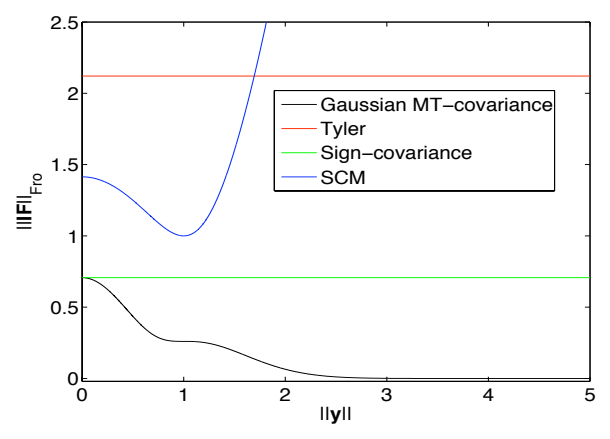

Fig. 1. Frobenius norms of the influence functions associated with the empirical Gaussian MT-covariance for $\tau=1$, Tyler's scatter Mestimator, the empirical sign-covariance, and the SCM, versus the contamination norm, for a bivariate standard complex normal distribution. Notice that the influence function approaches zero for large $\|\mathbf{y}\|$ only for the proposed Gaussian MT-covariance estimator.

\subsection{The Gaussian MT-covariance for compound Gaussian noise}

We assume that the noise component in (1) is compound Gaussian with the following stochastic representation

$$
\mathbf{W}_{n}=\nu_{n} \mathbf{Z}_{n},
$$

where $\nu_{n} \in \mathbb{R}_{+}^{*}$ is a first-order stationary process, and $\mathbf{Z}_{n} \in \mathbb{C}^{p}$ is a proper-complex wide-sense stationary Gaussian process with zeromean and unit covariance, which is statistically independent of $\nu_{n}$. Using (3), (5), (11) and (12), the Gaussian MT-covariance of $\mathbf{X}_{n}$ under the model (1) has the following structure:

$$
\boldsymbol{\Sigma}_{\mathbf{X}}^{\left(u_{\mathrm{G}}\right)}(\tau)=\mathbf{A}(\boldsymbol{\theta}) \boldsymbol{\Sigma}_{\alpha^{2} \mathbf{S}}^{(g)}(\tau) \mathbf{A}^{H}(\boldsymbol{\theta})+\sigma_{\alpha \mathbf{W}}^{2(h)}(\tau) \mathbf{I},
$$

where $\boldsymbol{\Sigma}_{\alpha^{2} \mathbf{S}}^{(g)}(\tau)$ is the covariance of the scaled signal component $\alpha_{n}^{2} \mathbf{S}_{n}, \alpha_{n} \triangleq \sqrt{\tau^{2} /\left(\tau^{2}+\nu_{n}^{2}\right)}$, under the transformed joint 
probability measure $Q_{\alpha, \mathbf{S}}^{(g)}$ with the MT-function $g(\alpha, \mathbf{S} ; \tau) \triangleq$ $\left(\pi \tau^{2} / \alpha^{2}\right)^{-p} \exp \left(-\alpha^{2}\|\mathbf{A S}\|_{2}^{2} / \tau^{2}\right)$. Since $g(\cdot, \cdot ; \cdot)$ is strictly positive and $\alpha_{n}^{2} \mathbf{S}_{n}$ has a non-singular covariance, by Property 3 in Proposition 1 the MT-covariance $\boldsymbol{\Sigma}_{\alpha^{2} \mathbf{S}}^{(g)}(\tau)$ must be non-singular. The term $\sigma_{\alpha \mathbf{W}}^{2(h)}(\tau)$ that multiplies the identity matrix $\mathbf{I}$ in the second summand of (13) is the variance of the scaled noise component $\alpha_{n} \mathbf{W}_{n}$ under the transformed joint probability measure $Q_{\alpha, \mathbf{W}}^{(h)}$ with the MT-function $h(\alpha ; \tau)=\mathrm{E}\left[g(\alpha, \mathbf{S} ; \tau) ; P_{\mathbf{S}}\right]$.

Thus, using the structure (13), it can be shown that since $\mathbf{A}(\boldsymbol{\theta})$ has full column rank, and $\boldsymbol{\Sigma}_{\alpha^{2} \mathbf{S}}^{(g)}(\tau)$ is non-singular, the $p-q$ smallest eigenvalues of $\boldsymbol{\Sigma}_{\mathbf{X}}^{\left(u_{\mathrm{G}}\right)}(\tau)$ are equal to $\sigma_{\alpha \mathrm{W}}^{2(h)}(\tau)$ and their corresponding eigenvectors span the null space of $\mathbf{A}^{H}(\boldsymbol{\theta})$.

\subsection{The Gaussian MT-MUSIC algorithm}

The empirical Gaussian MT-covariance is B-robust, and, under the compound Gaussian noise assumption (12), the noise subspace can be determined from the Gaussian MT-covariance eigendecomposition. The Gaussian MT-MUSIC algorithm is implemented by replacing the MT-function in (10) with the Gaussian MT-function (11).

\subsection{Choosing the scale parameter of the Gaussian MT-function}

While de-emphasizing non-informative outliers, e.g., caused by heavy-tailed distributions, the empirical Gaussian MT-covariance is less informative than the standard sample-covariance when the observations are normally distributed. This is seen in the following proposition that follows from the Gaussian Fisher information formula [22], Property 4 in Proposition 1, and elementary trace inequalities [23].

Proposition 4. Assume that the probability distribution $P_{\mathrm{x}}$ of the array outputs (1) is proper complex normal. The ratio between the Fisher information for estimating $\theta_{k} \in\left\{\theta_{1}, \ldots, \theta_{q}\right\}$ under the transformed probability measure $Q_{\mathrm{X}}^{\left(u_{\mathrm{G}}\right)}$ (with the MT-function (11)) and the corresponding Fisher information under $P_{\mathbf{X}}$ satisfy:

$$
\left(\frac{\tau^{2}}{\operatorname{tr}^{-1}\left[\boldsymbol{\Sigma}_{\mathbf{X}}^{-1}\right]+\tau^{2}}\right)^{2} \geq \frac{F\left(\theta_{k} ; Q_{\mathbf{x}}^{\left(u_{\mathrm{G}}\right)}\right)}{F\left(\theta_{k} ; P_{\mathbf{X}}\right)} \geq\left(\frac{\tau^{2}}{\operatorname{tr}\left[\boldsymbol{\Sigma}_{\mathbf{X}}\right]+\tau^{2}}\right)^{2}
$$

Therefore, in order to prevent a significant information loss when the observations are normally distributed, we propose to choose a safe-guard $\tau_{s}=\sqrt{c \operatorname{tr}\left[\boldsymbol{\Sigma}_{\mathbf{X}}\right]}$, where $c$ is some positive constant that guarantees that the Fisher information ratio (14) is greater than $(c /(1+c))^{2}$. Since in practice $\boldsymbol{\Sigma}_{\mathbf{X}}$ is unknown, its trace is replaced by an empirical estimate of the sum of variances.

\section{NUMERICAL EXAMPLE}

In this example, the performance of Gaussian MT-MUSIC is compared to the standard SCM-based MUSIC (SCM-MUSIC) [1] and to its robust generalizations based on the empirical sign-covariance (SGN-MUSIC) [7], and Tyler's scatter M-estimator (TYLERMUSIC) [9], [12]. We consider two independent 4-QAM signals with equal power $\sigma_{\mathrm{S}}^{2}$ impinging on a 16-element uniform linear array with $\lambda / 2$ spacing (Rayleigh resolution limit [24] of $7.162^{\circ}$ ) from DOAs $\theta_{1}=-2^{\circ}$ and $\theta_{2}=2^{\circ}$. Two noise scenarios are tested: (1) Zero-mean proper complex Gaussian noise with scaled identity covariance $\sigma_{\mathrm{W}}^{2} \mathbf{I}$, and (2) complex Cauchy noise with i.i.d. margins having zero location parameter and dispersion $\sigma_{\mathrm{W}}^{2}$. In both settings $N=1000$ snapshots were used. Following the approach proposed in subsection 3.4, the scale parameter $\tau$ of the
MT-function (11) is set to $\tau=\sqrt{\sum_{k=1}^{p} \hat{\sigma}_{X_{k}}^{2}}$, where $\hat{\sigma}_{X_{k}}^{2}=$ $\gamma^{-2}\left[\left(\operatorname{IQR}\left(\left\{\operatorname{Re}\left(X_{k, n}\right)\right\}_{n=1}^{N}\right)\right)^{2}+\left(\operatorname{IQR}\left(\left\{\operatorname{Im}\left(X_{k, n}\right)\right\}_{n=1}^{N}\right)\right)^{2}\right], \gamma \triangleq$ $1 /\left(2 \sqrt{2} \operatorname{erf}^{-1}(1 / 2)\right)$, is a robust interquartile range (IQR) estimate of variance. The generalized signal-to-noise-ratio (GSNR) is defined as GSNR $\triangleq 10 \log _{10} \sigma_{\mathbf{S}}^{2} / \sigma_{\mathbf{W}}^{2}$ and is used to index the performance shown in Fig. 2.

The RMSE performances versus GSNR and the pseudo-spectra corresponding to GSNR $=2[\mathrm{~dB}]$ averaged over 1000 Monte-Carlo simulations are depicted in Fig. 2. Note that for the Gaussian noise case all algorithms perform similarly. For the Cauchy noise case, the Gaussian MT-MUSIC outperforms all other robust MUSIC generalizations with significantly lower GSNR threshold region. This may be attributed to the fact that unlike the empirical sign-covariance and Tyler's scatter M-estimator, the influence function of the empirical Gaussian MT-covariance is negligible for large norm outliers (as illustrated in Fig. 1), which are likely in low GSNRs.
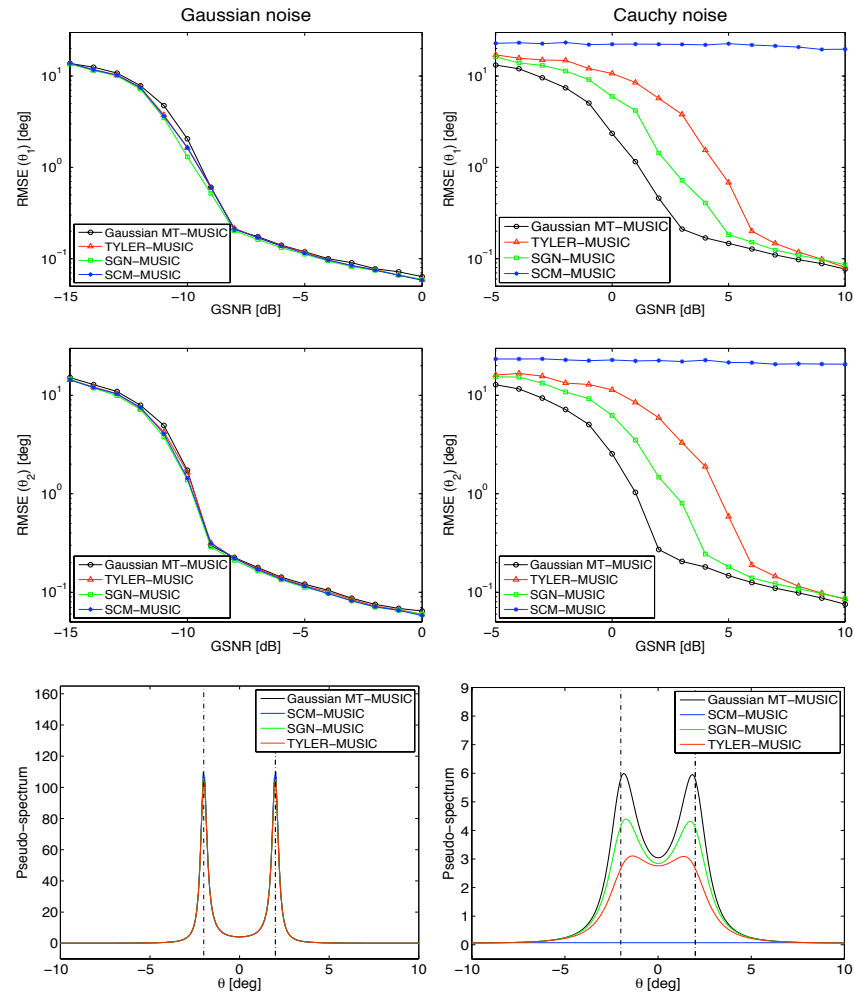

Fig. 2. RMSE for $\theta_{1}$ (top) and $\theta_{2}$ (middle) versus GSNR, and the pseudo-spectra corresponding to GSNR $=2[\mathrm{~dB}]$ (bottom) for the Gaussian (left) and Cauchy (right) noise scenarios. Note that for the Cauchy noise case, the proposed Gaussian MT-MUSIC estimator has lower MSE than the other methods, and its corresponding pseudospectrum has more highly resolved peaks at the true directions of arrival at angles $-2^{\circ}$ and $+2^{\circ}$.

\section{CONCLUSION}

In this paper a new framework for robust MUSIC was derived by applying a transform to the probability distribution of the data. Under the assumption of compound Gaussian noise, a new robust MUSIC algorithm, called Gaussian MT-MUSIC, was obtained by specifying the MT-function in the Gaussian family. Exploration of other classes of MT-functions may result in additional robust MUSIC algorithms. 


\section{REFERENCES}

[1] R. O. Schmidt, "Multiple emitter location and signal parameter estimation," IEEE Transactions on Antennas and Propagation, vol. 34, no. 3, pp. 276-280, 1986

[2] H. Krim, and M. Viberg, "Two decades of array signal processing research: the parametric approach," IEEE Signal Processing Magazine, vol. 13, no. 4, pp. 6794, 1996.

[3] P. G. Samorodnitsky and M. S. Taqqu, Stable non-Gaussian random processes: stochastic models with infinite variance. Chapman \& Hall, 1994.

[4] P. Tsakalides and C. L. Nikias, "The robust covariation-based MUSIC (ROCMUSIC) algorithm for bearing estimation in impulsive noise environments,' IEEE Transactions on Signal Processing, vol. 44, no. 7, pp. 1623-1633, 1996.

[5] C. L. Nikias and M. Shao, Signal processing with alpha-stable distributions and applications. Wiley-Interscience, 1995.

[6] T. H. Liu and J. M. Mendel, "A subspace-based direction finding algorithm using fractional lower order statistics," IEEE Transactions on Signal Processing, vol. 49, no. 8, pp. 1605-1613, 2001.

[7] S. Visuri, H. Oja, and V. Koivunen, "Subspace-based direction-of-arrival estimation using nonparametric statistics," IEEE Transactions on Signal Processing, vol. 49, no. 9, pp. 2060-2073, 2001

[8] F. R. Hampel, "The influence curve and its role in robust estimation," Journal of the American Statistical Association, vol. 69, no. 346, pp. 383-393, 1974.

[9] E. Ollila, D. E. Tyler, V. Koivunen, and H. V. Poor, "Complex elliptically symmetric distributions: survey, new results and applications," IEEE Transactions on Signal Processing, vol. 60, no. 1, pp. 5597-5625, 2012.

[10] R. A. Maronna, "Robust $M$-Estimators of Multivariate Location and Scatter," The Annals of Statistics, vol. 4, no. 1, pp. 51-67, 1976.

[11] P. J. Huber, Robust statistics. Wiley, 1981.

[12] D. E. Tyler, "A distribution-free M-estimator of multivariate scatter," Annals of statistics, vol. 15, no. 1, pp. 234-251, 1987.

[13] M. Hallin and D. Paindaveine, "Semiparametrically efficient rank-based inference for shape. I. Optimal rank-based tests for sphericity," The Annals of Statistics, vol. 34, no. 6, pp. 2707-2756, 2006.

[14] K. Todros and A. O. Hero, "On measure transformed canonical correlation analysis," IEEE Transactions on Signal Processing, vol. 60, no. 9, pp. 4570-4585, 2012

[15] K. Todros and A. O. Hero, "On measure independent component analysis," available online as arXiv:1302.0730.

[16] D. R. Cox, and D. V. Hinkley, Theoretical Statistics, p. 287, Chapman and Hall, 1974.

[17] E. Conte and M. Longo, "Characterization of radar clutter as a spherically invariant random process," IEE Proceedings F (Communications, Radar and Signal Processing), vol. 134, no. 2, pp. 191-197, 1987.

[18] F. Gini and A. Farina, "Vector subspace detection in compound-Gaussian clutter, part I: Survey and new results," IEEE Transactions on Aerospace and Electronic Systems, vol. 38, no. 4, pp. 1295-1311, 2002.

[19] M. Rangaswamy, "Spherically invariant random processes for modeling nonGaussian radar clutter," Proceeding of the 27th ASILOMAR Conference on Signals, Systems and Computers, pp. 1106-1110, 1993.

[20] E. Ollila, D. Tyler, V. Koivunen, and H. Poor, "Compound-Gaussian clutter modeling with an inverse Gaussian texture distribution" IEEE Signal Processing Letters, vol. 19, pp. 876-879, 2012.

[21] G. B. Folland, Real Analysis. John Wiley and Sons, 1984.

[22] P. Schreier and L. L. Scharf, Statistical signal processing of complex-valued data: the theory of improper and noncircular signals. p. 165, Cambridge University Press, 2010.

[23] R. Patel and M. Toda, "Trace inequalities involving Hermitian matrices," Linear algebra and its applications, vol. 23, pp. 13-20, 1979.

[24] H. L. Van Trees, Detection, Estimation, and Modulation Theory, Optimum Array Processing, p. 48, John Wiley \& Sons, 2004. 